\title{
28 Resarch Soure \\ Chronic lymphocytic leukemia; an incidental finding in a COVID-19 patient.
}

Shazaf Masood Sidhu ( $\nabla$ shazafmasood@gmail.com )

Aga Khan University Hospital

\section{Fabiha Ghulam Muhammad}

Aga Khan University Hospital

Ainan Arshad

Aga Khan University Hospital

\section{Case Report}

Keywords: Tumor lysis syndrome, Chronic Lymphocytic leukemia, Acute Kidney Injury, Incidental Finding, COVID-19 patient

Posted Date: July 30th, 2021

DOl: https://doi.org/10.21203/rs.3.rs-763505/v1

License: (c) (i) This work is licensed under a Creative Commons Attribution 4.0 International License. Read Full License 


\section{Abstract}

We report a case of 57 years old male, with no prior comorbids functional class I, presented with a history of fever for one week along with shortness of breath and cough for 5 days. Upon workup his baseline $\mathrm{CBC}$ reported bicytopenia along with marked lymphocytosis which raised the suspicion and to confirm the diagnosis, his acute leukemia comprehensive panel was done which reported an incidental finding of Chronic lymphocytic leukemia along with concomitant COVID PCR positive. This patient also presented with Tumor Lysis Syndrome and Acute kidney Injury.

\section{Background}

We report a case of 57 years old male, with no prior comorbids functional class I, presented with a history of fever for one week along with shortness of breath and cough for 5 days. Upon workup his baseline $\mathrm{CBC}$ reported bicytopenia along with marked lymphocytosis which raised the suspicion and to confirm the diagnosis, his acute leukemia comprehensive panel was done which reported an incidental finding of Chronic lymphocytic leukemia along with concomitant COVID PCR positive. This patient also presented with Tumor Lysis Syndrome and Acute kidney Injury.

\section{Case Presentation}

57 year gentleman with no prior comorbid, resident of Larkana, Sind presented with the complains of fever for one week. He was given antipyretics at home, fever did not resolve and he started developing shortness of breath for 2 days thus he was brought to Aga Khan University Hospital for further management on 27th april'2021. In the emergency department his vitals were: Blood pressure 121/73, Pulse:80beats/minute, Temperature 36.8 Celcius, Respiratory rate 27 breaths per minute, Oxygen saturation $90 \%$ on room air. On physical examination he was tachypneac, and had bilateral basal crepitations on auscultation.

SARS CoV-2(antigen) was checked that came out to be positive. Upon seeing raised TLC

He was admitted in special care unit and was managed on the lines of Critical Covid Pnumonia, CLL with tumor lysis syndrome and acute kidney injury.

\section{INVESTIGATIONS}




\begin{tabular}{|c|c|c|}
\hline Laboratory tests & On admission (27.04.2021) & \\
\hline Hemoglobin (gm/dl) & 12.3 & \\
\hline Total Leucocyte count ${ }^{\star} 10^{9}$ & 111.5 & \\
\hline Lymphocytes \% & 95.5 & \\
\hline Neutrophils \% & 3.5 & \\
\hline Platelets $* 10^{9}$ & 59 & \\
\hline Calcium (mg/dl) & 8.5 & \\
\hline Uric Acid (mg/dl) & 7.8 & \\
\hline BUN (mg/dl) & 22 & \\
\hline Creatinine (mg/dl) & 2.2 & \\
\hline Sodium (mmol/L) & 138 & \\
\hline Potassium (mmol/L) & 4.5 & \\
\hline Chloride (mmol/L) & 98 & \\
\hline Bicarbonate (mmol/L) & 19.8 & \\
\hline CRP (mg/L) & 123 & \\
\hline Ferritin (ng/ml) & 383 & \\
\hline Pro BNP $(\mathrm{pg} / \mathrm{mL})$ & 183 & \\
\hline LDH (IU/L) & 456 & \\
\hline D-Dimer (mg/L FEU) & 1.2 & \\
\hline SGPT (IU/L) & 40 & \\
\hline SGOT (IU/L) & 69 & \\
\hline Interleukin-6 level (pg/ml) & & 165 \\
\hline TSH (ulU/ml) & & 0.120 \\
\hline FT4 (ng/dl) & & 1.16 \\
\hline
\end{tabular}

Peripheral Blood Smear showed: Anisocytosis, Lymphocytosis and smear cells.

Immune phenotyping was sent that showed Mature B-Cell Lymphoproliferative disorder (B-CLL) 
First differential diagnosis just based on clinical examination was pulmonary edema, but ECG was normal, Troponin and Pro BNP was negative,

Second differential diagnosis in middle aged with AKI and hypercalcemia was multiple myeloma but patient lacked anemia and there was no hypercalcemia.

\section{TREATMENT}

He was started on treatment for Covid pneumonia, admitted to special care unit, supplemental oxygen given via nasal cannula, Non-Invasive ventilator support was applied for work of breathing. Hematology was taken on board and they agreed for starting steroids as per Covid management. He was started on IV.Dexammethasone $6 \mathrm{mg}$ twice daily, IV. Remdesivir was given for total 5 days, Unfractionated Heparin $5000 \mathrm{mg}$ subcutaneously was given twice daily. Intermittent diuresis was done depending on volume status to avoid developing ARDS. Antibiotic coverage was given considering functional deficiency might lead to full blown sepsis. Initially patient's oxygen and NIV requirement tailored down but after 2 days he deteriorated and developed increasing oxygen demands, His Interleukin-6 levels were done that were raised hence he was commenced on IV.Tocilizumab after discussing with Infectious Disease and Hematology teams. No further treatment for CLL was initiated at the moment. Patient also had subclinical hyperthyroidism that was attributable to current medical condition and initiated on any antithyroid drugs for now.

\section{OUTCOME AND FOLLOW-UP}

Currently patient is still under treatment requiring oxygen support and NIV.

His condition is critical as his leukocytosis are in increasing trend and hypoxia is not improving. Although his AKI has resolved and patient is making adequate urine output. His GCS is full and other systemic examination is normal for now.

\section{Discussion}

Patients with cancer are prone to develop Covid-19 due to immune deficiency state and chemotherapeutic effects ${ }^{(2)}$. It is important to analyze slight change in health and daily activities which should prompt thorough investigation so that major diseases are not missed. Patients with an immunocompromised state typically CLL have poor prognosis with concomitant COVID-19 and are more prone to superimposed bacterial infections which can cause sequel of complications. But an early detection can help with a better treatment plan after recovery from COVID illness

\section{LEARNING POINTS/TAKE HOME MESSAGES}

- Differential diagnosis should be thoroughly discussed and investigated before any diagnosis is finalized

- Multidisciplinary approach should be opted while dealing with complicated cases. 
- Active researches should be carried out to have guidelines for managing high stake comorbidites in Covid.

- Cancer treatment should not be halted in Covid pandemic

\section{References}

- Wang D, Hu B, Hu C, et al. Clinical Characteristics of 138 Hospitalized Patients with 2019 Novel Coronavirus-Infected Pneumonia in Wuhan, China. JAMA. 2020;1323(11):1061-69. [PubMed] [Google Scholar]

- Scarfò L, Chatzikonstantinou T, Rigolin GM, Quaresmini G, Motta M, Vitale C, Garcia-Marco JA, Hernández-Rivas JÁ, Mirás F, Baile M, Marquet J. COVID-19 severity and mortality in patients with chronic lymphocytic leukemia: a joint study by ERIC, the European Research Initiative on CLL, and CLL Campus. Leukemia. 2020 Sep;34(9):2354-63.

\section{Declarations}

Source of funding: None

Conflict of interest: None

The patient gave written consent to participate. ERC approval was obtained as well (ERC number: 20216308-17930).

\section{Figures}




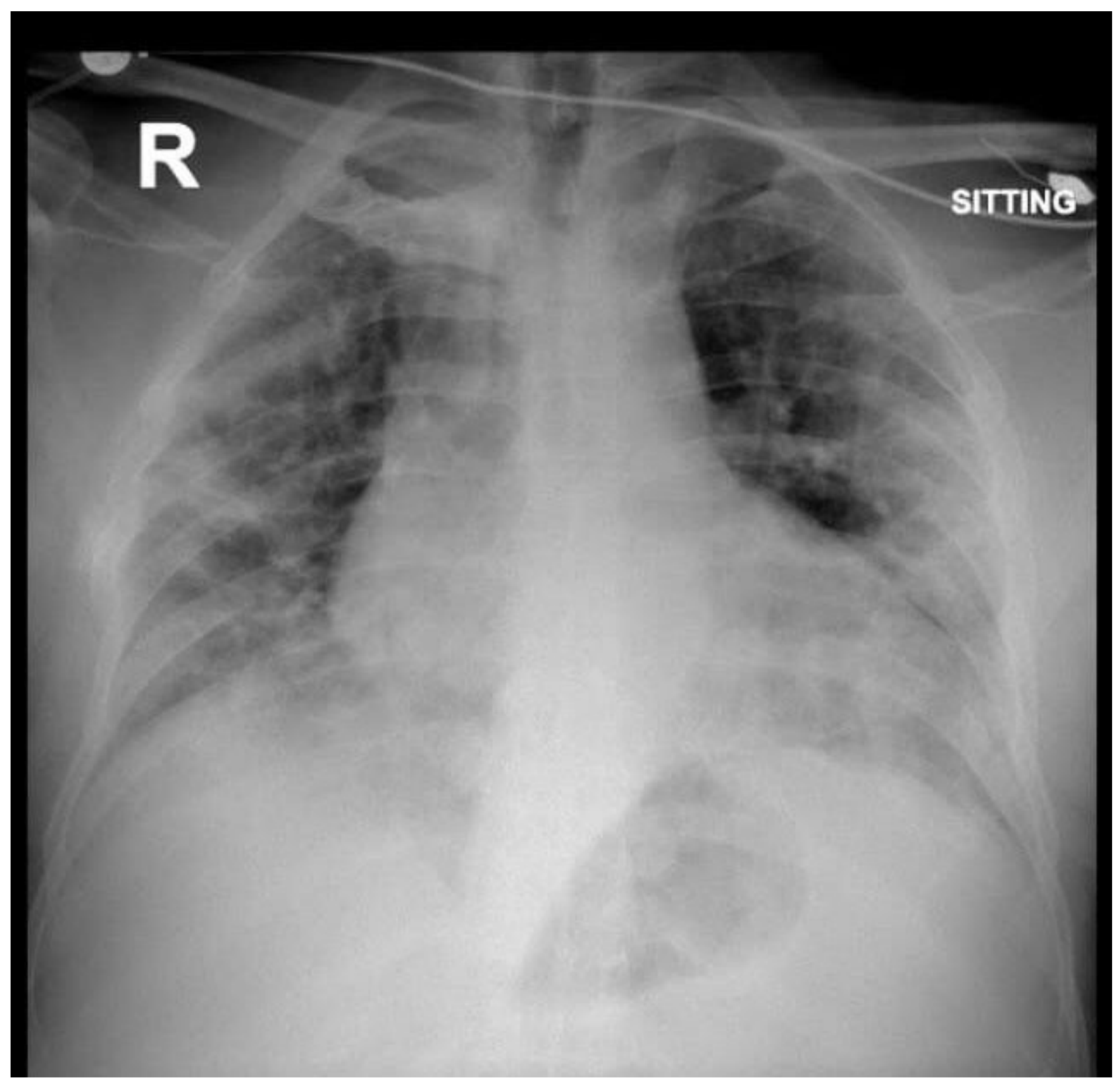

Figure 1

Chest XRAY AP view on admission. 